\title{
A CONFLUENT HYPERGEOMETRIC INTEGRAL EQUATION
}

\author{
by E. R. LOVE, T. R. PRABHAKAR and N. K. KASHYAP
}

(Received 19 May, 1980)

1. Introduction. Recently there have appeared papers ([7], [8]; also see [9]) in which integral equations with kernels involving the confluent hypergeometric function

$$
{ }_{1} F_{1}(a ; c ; z)=\sum_{n=0}^{\infty} \frac{(a)_{n}}{(c)_{n}} \frac{z^{n}}{n !}, \text { where }(a)_{n}=\frac{\Gamma(a+n)}{\Gamma(a)},
$$

have been studied. These equations are mainly Volterra equations of the first kind except that they have infinite domain $(0, \infty)$. The rest are of the related type with integrals over $(x, \infty)$ instead of $(0, x)$; and all are convolution equations.

The equation solved in this paper is a Fredholm equation of the first kind except for infinite domain:

$$
\int_{0}^{\infty} \frac{t^{c-1}}{\Gamma(c)}{ }_{1} F_{1}(a ; c ;-x t) f(t) d t=\frac{g(x)}{\Gamma(a)} \text { for all } x>0,
$$

where $f$ is the unknown function and the parameters $a$ and $c$ have positive real parts. Formally the relationship of this equation to those in [7] and [8] is similar to that of the equation in [5] to those in [3] and [4]. However, the equations in [3], [4] and [5] have Gauss's hypergeometric function ${ }_{2} F_{1}$ in place of the confluent function.

Preliminary work on the Weyl fractional integral and derivative is set out in $\S \S 2$ and 3 . This augments the treatments given in [4] and [6], neither of which is adequate for the present purpose.

2. Weyl Fractional Integrals. We use the customary definition

$$
J^{\nu} f(x)=\int_{x}^{\infty} \frac{(t-x)^{\nu-1}}{\Gamma(\nu)} f(t) d t=\int_{0}^{\infty} \frac{t^{\nu-1}}{\Gamma(\nu)} f(x+t) d t,
$$

where re $\nu>0$ and the integral is Lebesgue. But, following Lighthill [3] and Miller [5], we restrict $f$ to belong to a class $E$ defined by:

(a) $f$ is a complex-valued infinitely differentiable function on $(0, \infty)$,

(b) $x^{k} f^{(r)}(x) \rightarrow 0$ as $x \rightarrow \infty$ for each fixed $k$ and $r, r \geq 0$.

Thus if $f \in E$ and $n$ is a positive integer, then $f^{(n)} \in E$.

Lemma 1. If $f \in E$, re $\nu>0, n$ is a positive integer and $D=d / d x$, then $J^{\nu} f(x), D^{n} J^{\nu} f(x)$ and $J^{\nu} D^{n} f(x)$ exist for all $x>0$ and

$$
D^{n} J^{\nu} f(x)=J^{\nu} D^{n} f(x) \text {. }
$$

Proof. (i) For fixed $[a, b] \subset(0, \infty), f$ is continuous in $[a, b+1]$; so

$$
\left|t^{\nu-1} f(x+t)\right| \leq M t^{\mathrm{re \nu}-1} \text { for } a \leq x \leq b \text { and } 0<t \leq 1 \text {. }
$$

Glasgow Math. J. 23 (1982) 31-40. 
The improper integral

$$
\int_{\rightarrow 0}^{1} t^{\nu-1} f(x+t) d t
$$

is therefore absolutely and uniformly convergent on $a \leq x \leq b$.

A similar argument applies if a derivative $f^{(r)}$ replaces $f$. So

$$
\frac{d}{d x} \int_{\rightarrow 0}^{1} t^{\nu-1} f^{(r-1)}(x+t) d t=\int_{\rightarrow 0}^{1} t^{\nu-1} f^{(r)}(x+t) d t
$$

for $a<x<b$, and consequently for all $x>0$.

(ii) There is $T>1$ such that $\left|s^{\text {rev }+1} f(s)\right|<1$ for all $s \geq T$. So

$$
\left|t^{\nu-1} f(x+t)\right|=t^{-2} t^{\mathrm{re} \nu+1}|f(x+t)| \leq t^{-2}(x+t)^{\mathrm{re} \nu+1}|f(x+t)|<t^{-2}
$$

whenever $x \geq 0$ and $t \geq T$. So the infinite integral

$$
\int_{1}^{\rightarrow \infty} t^{\nu-1} f(x+t) d t
$$

is absolutely and uniformly convergent on $x \geq 0$. Similarly when $f$ is replaced by $f^{(r)}$. Thus as in (i) we obtain, for all $x>0$,

$$
\frac{d}{d x} \int_{1}^{\rightarrow \infty} t^{\nu-1} f^{(r-1)}(x+t) d t=\int_{1}^{\rightarrow \infty} t^{\nu-1} f^{(r)}(x+t) d t .
$$

(iii) These integrals, being absolutely convergent, can be replaced by Lebesgue integrals. Thus we have existence of $J^{\nu} f(x)$, and

$$
\frac{d}{d x} \int_{0}^{\infty} t^{\nu-1} f^{(r-1)}(x+t) d t=\int_{0}^{\infty} t^{\nu-1} f^{(r)}(x+t) d t
$$

for all $x>0$, and the lemma follows.

THEOREM 2. If re $\nu>0$ and $f \in E$ then $J^{\nu} f \in E$.

Proof. Requirement (a) for $J^{\nu} f$ to be in $E$ follows from Lemma 1. To prove that requirement (b) is satisfied, it is enough to consider positive $k$. Given $k>0$ and $\epsilon>0$, there is $X>0$ such that

$$
\begin{aligned}
x^{k+\mathrm{re} \nu+1} \mid & |f(x)|<\epsilon \text { whenever } x>X . \\
x^{k}\left|\int_{0}^{\infty} t^{\nu-1} f(x+t) d t\right| & \leq \int_{0}^{\infty} \frac{\left|t^{\nu-1}\right|}{(x+t)^{\mathrm{re} \nu+1}}(x+t)^{k+\mathrm{re} \nu+1}|f(x+t)| d t \\
& \leq \int_{0}^{\infty} \frac{t^{\mathrm{rev}-1}}{(x+t)^{\mathrm{re} \nu+1}} \epsilon d t \text { if } x>X, \\
& =\frac{\epsilon}{x} \int_{0}^{\infty} \frac{u^{\mathrm{rev}-1}}{(1+u)^{\mathrm{re} \nu+1}} d u \text { by } t=x u, \\
& <\epsilon \text { if } x>X+\int_{0}^{\infty} \frac{u^{\mathrm{re} \nu-1}}{(1+u)^{\mathrm{re} \nu+1}} d u,
\end{aligned}
$$

this integral being convergent. Thus $x^{k} J^{v} f(x) \rightarrow 0$ as $x \rightarrow \infty$. 
Similarly with $f$ replaced by $f^{(r)}$. So, using Lemma 1 ,

$$
x^{k} D^{r} J^{\nu} f(x)=x^{k} J^{\nu} D^{r} f(x) \rightarrow 0 \text { as } x \rightarrow \infty .
$$

THEOREM 3. If re $\mu>0$, re $\nu>0$ and $f \in E$, then for all $x>0$

$$
J^{\nu} J^{\mu} f(x)=J^{\mu+\nu} f(x) \text {. }
$$

Proof. By Theorem 2, $J^{\mu} f \in E, J^{\nu} J^{\mu} f \in E$, and $J^{\mu+\nu} f \in E$; so both sides of the desired equation exist for all $x>0$.

$$
\begin{aligned}
\Gamma(\mu) \Gamma(\nu) J^{\nu} J^{\mu} f(x) & =\Gamma(\mu) \int_{0}^{\infty} t^{\nu-1} J^{\mu} f(t+x) d t \\
& =\int_{0}^{\infty} t^{\nu-1} d t \int_{0}^{\infty} s^{\mu-1} f(s+t+x) d s \\
& =\int_{0}^{\infty} t^{\nu-1} d t \int_{t}^{\infty}(u-t)^{\mu-1} f(u+x) d u \\
& =\int_{0}^{\infty} f(u+x) d u \int_{0}^{u}(u-t)^{\mu-1} t^{\nu-1} d t \\
& =\frac{\Gamma(\mu) \Gamma(\nu)}{\Gamma(\mu+\nu)} \int_{0}^{\infty} u^{\mu+\nu-1} f(x+u) d u ;
\end{aligned}
$$

this proves the theorem provided that the change of order of integration in the second-last step is justified. For this we prove absolute convergence of the repeated integral as follows. Let $g(s)=|f(s)|$. We have

$$
\begin{aligned}
\int_{0}^{\infty}|f(u+x)| d u \int_{0}^{u}\left|(u-t)^{\mu-1} t^{\nu-1}\right| d t & =\int_{0}^{\infty} g(u+x) d u \int_{0}^{u}(u-t)^{\mathrm{re} \mu-1} t^{\mathrm{re} \nu-1} d t \\
& =\frac{\Gamma(\mathrm{re} \mu) \Gamma(\mathrm{re} \nu)}{\Gamma(\mathrm{re} \mu+\mathrm{re} \nu)} \int_{0}^{\infty} u^{\mathrm{re} \mu+\mathrm{re} \nu-1} g(x+u) d u .
\end{aligned}
$$

To prove the last integral convergent, we have that $g(x+u)$ is a continuous function of $u$ in $(-x, \infty)$, and so in $[0, \infty)$ since $x>0$. So $g(x+u)$ is bounded on $0 \leq u \leq 1$, and the last integral is convergent at the lower terminal. It is also convergent at the upper terminal because, for fixed $x>0$,

$$
u^{\mathrm{re} \mu+\mathrm{rev}-1} \mathrm{~g}(x+u) \leq u^{-2}(x+u)^{\mathrm{re} \mu+\mathrm{re} \nu+1}|f(x+u)|=o\left(u^{-2}\right) \quad \text { as } \quad u \rightarrow \infty .
$$

This proves the required absolute convergence.

LemMA 4. If $f \in E$, re $\nu>0, n$ is a positive integer and $D=d / d x$, then for all $x>0$

$$
(-D)^{n} J^{\nu+n} f(x)=J^{\nu} f(x)
$$

Proof. This is obvious for $n=0$, the existence being assured by Lemma 1. Assume it true for $n=1, \ldots, r$. The $n$th derivative exists for all $n$ by Lemma 1, and by Theorem 3

$$
(-D)^{r+1} J^{\nu+r+1} f(x)=(-D)^{r+1} J^{\nu+r} J^{1} f(x)=(-D) J^{\nu} J^{1} f(x)
$$


by the assumed case $n=r$, since $J^{1} f \in E$ by Theorem 2 . So, by Theorem 3 again, and then by the assumed case $n=1$,

$$
(-D)^{r+1} J^{\nu+r+1} f(x)=-D J^{\nu+1} f(x)=J^{\nu} f(x), \text { as required. }
$$

3. Weyl Fractional Derivatives. Our definition of ath derivative is suggested by Lemma 4 ; it is

$$
J^{-a} f(x)=(-D)^{n} J^{n-a} f(x),
$$

where re $a \geq 0$ and $n$ is any integer such that $n>$ re $a$.

The right side exists for each $x>0$ and integer $n>$ re $a$, by Lemma 1 or Theorem 2 . But we need to prove consistency-that it is the same for all such $n$.

LEMmA 5. If $f \in E$, re $a \geq 0$ and $x>0$ then $(-D)^{n} J^{n-a} f(x)$ is the same for all integers $n>$ re $a$; and $J^{-a} f \in E$.

Proof. (i) Let $m$ be the least such integer $n$, and let $n$ be any integer greater than $m$. Then by Lemma 4 with $\nu$ and $n$ replaced by $m-a$ and $n-m$,

$$
(-D)^{n} J^{n-a} f(x)=(-D)^{m}(-D)^{n-m} J^{n-a} f(x)=(-D)^{m} J^{m-a} f(x) .
$$

(ii) Using the definition and Lemma 1,

$$
J^{-a} f(x)=(-D)^{n} J^{n-a} f(x)=(-1)^{n} J^{n-a} D^{n} f(x) .
$$

Since $D^{n} f \in E$, Theorem 2 gives that $J^{n-a} D^{n} f \in E$; consequently $J^{-a} f \in E$, as required.

THEOREM 6. If $f \in E$ and $n$ is a positive integer or zero, then for all $x>0$ we have $J^{-n} f(x)=(-D)^{n} f(x)$.

Proof. For the case $n=0$ the definition gives

$$
J^{0} f(x)=-D J^{1} f(x)=-D \int_{x}^{\infty} f(t) d t=f(x) .
$$

For $n>0$ the definition, with $a$ and $n$ replaced by $n$ and $n+1$, gives

$$
\begin{aligned}
J^{-n} f(x) & =(-D)^{n+1} J^{(n+1)-n} f(x) \\
& =(-D)^{n}(-D) J^{1} f(x)=(-D)^{n} f(x),
\end{aligned}
$$

the last step using the calculation made in (4).

Lemma 7. If re $a \geq 0$, re $b \geq 0$ and $f \in E$, then for all $x>0$

$$
J^{-b} J^{-a} f(x)=J^{-a-b} f(x) \text {. }
$$

Proof. Let $m$ and $n$ be positive integers such that $m>\operatorname{re} a$ and $n>\operatorname{re} b$. By the 
definition, and (3),

$$
\begin{aligned}
J^{-b} J^{-a} f & =(-D)^{n} J^{n-b} J^{-a} f \\
& =(-D)^{n} J^{n-b} J^{m-a}(-D)^{m} f \\
& =(-D)^{n} J^{m+n-a-b}(-D)^{m} f \\
& =(-D)^{n}(-D)^{m} J^{m+n-a-b} f \\
& =(-D)^{m+n} J^{m+n-a-b} f=J^{-a-b} f .
\end{aligned}
$$

For (5) we have used Theorem 3 and the fact that $(-D)^{m} f \in E$. For (6) we have used Lemma 1 . The first and last steps use Lemma 5 implicitly.

THEOREM 8. If $a$ and $b$ are any complex numbers, and $f \in E$, then for all $x>0$ we have $J^{b} J^{a} f(x)=J^{a+b} f(x)$.

Proof. (i) Suppose that re $a \leq 0<$ re $b$ and let $m$ be an integer such that $m>\operatorname{re}(-a)$. By Theorem 2, $J^{m+a} f \in E$; so, by definition, Lemma 1 and Theorem 3,

$$
J^{b} J^{a} f=J^{b}(-D)^{m} J^{m+a} f=(-D)^{m} J^{b} J^{m+a} f=(-D)^{m} J^{m+a+b} f .
$$

If $\mathrm{re}(a+b)>0$ the last expression is equal to $J^{a+b} f$, by Lemma 4 ; while if $\operatorname{re}(a+b) \leq 0$ the same is true by definition, since $m>\operatorname{re}(-a)>\operatorname{re}(-a-b)$.

(ii) Suppose that re $a>0 \geq \mathrm{re} b$, and let $n$ be an integer such that $n>\mathrm{re}(-b)$. By definition and Theorem 3,

$$
J^{b} J^{a} f=(-D)^{n} J^{n+b} J^{a} f=(-D)^{n} J^{n+b+a} f
$$

If re $(a+b)>0$ the last expression is equal to $J^{a+b} f$ by Lemma 4 ; while if re $(a+b) \leq 0$ the same is true by definition, since $n>\operatorname{re}(-b)>\operatorname{re}(-a-b)$.

(iii) The remaining cases are covered by Theorem 3 and Lemma 7:

4. An Integral Transform. The transform occurring in our integral equation involves the confluent hypergeometric function ${ }_{1} F_{1}$, defined by

$$
{ }_{1} F_{1}(a ; c ; z)=\sum_{n=0}^{\infty} \frac{(a)_{n}}{(c)_{n}} \frac{z^{n}}{n !}
$$

for all complex $a, c, z$ with $c \neq 0,-1,-2, \ldots$ As usual $(a)_{0}=1$ and

$$
(a)_{n}=a(a+1)(a+2) \ldots(a+n-1)=\frac{\Gamma(a+n)}{\Gamma(a)} .
$$

Lemma 9. If $a, c, k, z$ are complex, re $k>$ re $c>0$ and $t>0$, then

$$
\int_{0}^{t} \frac{(t-s)^{k-c-1}}{\Gamma(k-c)} \frac{s^{c-1}}{\Gamma(c)}{ }_{1} F_{1}(a ; c ; z s) d s=\frac{t^{k-1}}{\Gamma(k)}{ }_{1} F_{1}(a ; k ; z t) .
$$


Proof. Provided the term by term integration at (9) is correct, the left side is equal to

$$
\begin{aligned}
& \sum_{n=0}^{\infty} \frac{(a)_{n}}{(c)_{n}} \frac{z^{n}}{n !} \int_{0}^{t} \frac{(t-s)^{k-c-1}}{\Gamma(k-c)} \frac{s^{n+c-1}}{\Gamma(c)} d s \\
= & \sum_{n=0}^{\infty}(a)_{n} \frac{z^{n}}{n !} \int_{0}^{t} \frac{(t-s)^{k-c-1}}{\Gamma(k-c)} \frac{s^{c+n-1}}{\Gamma(c+n)} d s \\
= & \sum_{n=0}^{\infty}(a)_{n} \frac{z^{n}}{n !} \frac{t^{k+n-1}}{\Gamma(k+n)}=\frac{t^{k-1}}{\Gamma(k)} \sum_{n=0}^{\infty} \frac{(a)_{n}}{(k)_{n}} \frac{z^{n} t^{n}}{n !}=\frac{t^{k-1}}{\Gamma(k)}{ }_{1} F_{1}(a ; k ; z t) .
\end{aligned}
$$

To justify the term by term integration it is enough to show that (9), or equally (10), is convergent when every factor is replaced by its modulus. For this, write $\alpha, \gamma, \kappa$ for the real parts of $a, c, k$; then

$$
\begin{aligned}
\left|(a)_{n} \frac{z^{n}}{n !}\right| \int_{0}^{t}\left|\frac{(t-s)^{k-c-1}}{\Gamma(k-c)} \frac{s^{c+n-1}}{\Gamma(c+n)}\right| d s & \begin{aligned}
= & \left|(a)_{n}\right| \frac{|z|^{n}}{n !} \frac{\Gamma(\kappa-\gamma)}{|\Gamma(k-c)|} \frac{\Gamma(\gamma+n)}{|\Gamma(c+n)|} \int_{0}^{1} \frac{(t-s)^{\kappa-\gamma-1}}{\Gamma(\kappa-\gamma)} \frac{s^{\gamma+n-1}}{\Gamma(\gamma+n)} d s \\
& =\left|\frac{\Gamma(a+n)}{\Gamma(a)}\right| \frac{|z|^{n}}{n !} \frac{\Gamma(\kappa-\gamma)}{\mid \Gamma(k-c)} \frac{\Gamma(\gamma+n)}{|\Gamma(c+n)|} \frac{t^{\kappa+n-1}}{\Gamma(\kappa+n)} \\
& =O\left(n^{\alpha-\kappa}|z t|^{n} / n !\right) .
\end{aligned}
\end{aligned}
$$

This proves the required convergence, and so establishes the lemma. The restriction that $\kappa>\gamma>0$ ensures convergence of the integral in (10), and is also used similarly in the justification.

THEOREM 10. If $a, c, k$ are complex, re $k>\operatorname{re} c>0, x>0, f \in E$ and $t^{k-1} f(t) \in L(0,1)$, then

$$
\int_{0}^{\infty} \frac{t^{k-1}}{\Gamma(k)}{ }_{1} F_{1}(a ; k ;-x t) f(t) d t=\int_{0}^{\infty} \frac{t^{c-1}}{\Gamma(c)}{ }_{1} F_{1}(a ; c ;-x t) J^{k-c} f(t) d t .
$$

Proof. Using Lemma 9 with $z=-x$, the left side is formally

$$
\begin{aligned}
& \int_{0}^{\infty} f(t) d t \int_{0}^{t} \frac{(t-s)^{k-c-1}}{\Gamma(k-c)} \frac{s^{c-1}}{\Gamma(c)}{ }_{1} F_{1}(a ; c ;-x s) d s \\
= & \int_{0}^{\infty} \frac{s^{c-1}}{\Gamma(c)}{ }_{1} F_{1}(a ; c ;-x s) d s \int_{s}^{\infty} \frac{(t-s)^{k-c-1}}{\Gamma(k-c)} f(t) d t
\end{aligned}
$$

and this by (1) is equal to the right side. It remains only to prove the existence and equality of (11) and (12); and these are assured if we prove the absolute convergence of (11). The inner integral in (12) exists a.e. by this argument, but everywhere by Lemma 1 .

By $[1: 6.13(3)]$, and by continuity,

$$
\left.\begin{array}{lll}
{ }_{1} F_{1}(a ; c ;-x s)=O\left((x s)^{-a}\right) & \text { for } & x s>1, \\
{ }_{1} F_{1}(a ; c ;-x s)=O(1) & \text { for } & |x s| \leq 1
\end{array}\right\}
$$


If re $a \geq 0$, this function is $O(1)$ for all $s>0$, and consequently the absolute integral corresponding to (11) is majorized by

$$
\int_{0}^{\infty}|f(t)| d t \int_{0}^{t} \frac{(t-s)^{\kappa-\gamma-1}}{\Gamma(\kappa-\gamma)} \frac{s^{\gamma-1}}{\Gamma(\gamma)} d s=\int_{0}^{\infty}|f(t)| \frac{t^{\kappa-1}}{\Gamma(\kappa)} d t,
$$

where $\gamma$ and $\kappa$ again denote the real parts of $c$ and $k$. The last integral is finite; for the part of it on $(0,1)$ is finite by hypothesis, and the part on $(1, \infty)$ is finite because

$$
f(t) t^{\kappa-1}=o\left(t^{-2}\right) \text { as } t \rightarrow \infty .
$$

Now suppose that $\alpha=$ re $a<0$. Write $m$ for $\min \{t, 1 / x\}$. The absolute integral corresponding to (11) is majorized, using (13), by

$$
\begin{aligned}
& \int_{0}^{\infty}|f(t)| d t \int_{0}^{m} \frac{(t-s)^{\kappa-\gamma-1}}{\Gamma(\kappa-\gamma)} \frac{s^{\gamma-1}}{\Gamma(\gamma)} d s+\int_{1 / x}^{\infty}|f(t)| d t \int_{m}^{t} \frac{(t-s)^{\kappa-\gamma-1}}{\Gamma(\kappa-\gamma)} \frac{s^{\gamma-1}}{\Gamma(\gamma)}(x s)^{-\alpha} d s \\
\leq & \int_{0}^{\infty}|f(t)| d t \int_{0}^{t} \frac{(t-s)^{\kappa-\gamma-1}}{\Gamma(\kappa-\gamma)} \frac{s^{\gamma-1}}{\Gamma(\gamma)} d s+x^{-\alpha} \frac{\Gamma(\gamma-\alpha)}{\Gamma(\gamma)} \int_{1 / x}^{\infty}|f(t)| d t \int_{0}^{t} \frac{(t-s)^{\kappa-\gamma-1}}{\Gamma(\kappa-\gamma)} \frac{s^{\gamma-\alpha-1}}{\Gamma(\gamma-\alpha)} d s \\
= & \int_{0}^{\infty}|f(t)| \frac{t^{\kappa-1}}{\Gamma(\kappa)} d t+x^{-\alpha} \frac{\Gamma(\gamma-\alpha)}{\Gamma(\gamma)} \int_{1 / x}^{\infty}|f(t)| \frac{t^{\kappa-\alpha-1}}{\Gamma(\kappa-\alpha)} d t .
\end{aligned}
$$

Of these two integrals, the former is convergent as in the preceding paragraph, and the latter as in (14), since $f \in E$ and so

$$
f(t) t^{\kappa-\alpha-1}=o\left(t^{-2}\right) \quad \text { as } \quad t \rightarrow \infty .
$$

Thus (11) is absolutely convergent and the proof is complete.

REMARK. The integrability hypothesis in Theorem 10 may seem a regrettable stray. But it is inevitable for the existence of the integral on the left of the theorem, since the integrand is asymptotic to $f(t) t^{k-1} / \Gamma(k)$ as $t \rightarrow 0$.

Lemma 11. If re $a>0$, re $c>0, f \in E$, and either

(i) re $c>$ re $a$ and $t^{c-1} f(t) \in L(0,1)$, or

(ii) re $a>$ re $c$ and $t^{a-1} J^{c-a} f(t) \in L(0,1)$, or

(iii) re $k>\max \{$ re $a$, re $c\}$ and $t^{k-1} J^{c-k} f(t) \in L(0,1)$, then for all $x>0$

$$
\int_{0}^{\infty} \frac{t^{c-1}}{\Gamma(c)}{ }_{1} F_{1}(a ; c ;-x t) f(t) d t=\int_{0}^{\infty} e^{-x t} \frac{t^{a-1}}{\Gamma(a)} J^{c-a} f(t) d t .
$$

Proof. (i) In Theorem 10 replace $k$ and $c$ by $c$ and $a$.

(ii) In Theorem 10 replace $k$ and $f$ by $a$ and $J^{c-a} f$. This fractional derivative exists in $E$ by Lemma 5 . The left side in Theorem 10 becomes the right side of (15); and the right side in Theorem 10 becomes

$$
\int_{0}^{\infty} \frac{t^{c-1}}{\Gamma(c)}{ }_{1} F_{1}(a ; c ;-x t) J^{a-c} J^{c-a} f(t) d t
$$


which is the left side of (15) by Theorem 8 (actually by case (i) of the proof of that theorem) and (4).

(iii) In Theorem 10 replace $f$ by $J^{c-k} f$, which exists in $E$ by Lemma 5. This gives

$$
\int_{0}^{\infty} \frac{t^{k-1}}{\Gamma(k)}{ }_{1} F_{1}(a ; k ;-x t) J^{c-k} f(t) d t=\int_{0}^{\infty} \frac{t^{c-1}}{\Gamma(c)}{ }_{1} F_{1}(a ; c ;-x t) f(t) d t
$$

because $J^{k-c} J^{c-k} f=J^{0} f=f$ by Theorem 8 (again by case (i) of its proof) and (4).

In Theorem 10 replace $c$ and $f$ by $a$ and $J^{c-k} f$. This gives

$$
\int_{0}^{\infty} \frac{t^{k-1}}{\Gamma(k)}{ }_{1} F_{1}(a ; k ;-x t) J^{c-k} f(t) d t=\int_{0}^{\infty} \frac{t^{a-1}}{\Gamma(a)} e^{-x t} J^{k-a} J^{c-k} f(t) d t .
$$

Equating the right sides of (16) and (17) we obtain (15), because $J^{k-a} J^{c-k} f=J^{c-a} f$ by Theorem 8.

Remark. Cases (i) and (ii) of Lemma 11 may be regarded as limiting cases of case (iii), with $k=c$ for case (i) and $k=a$ for case (ii). A similar remark may be made about Theorem 12.

5. Solutions of the Integral Equation. We seek functions $f$ satisfying

$$
\int_{0}^{\infty} \frac{t^{c-1}}{\Gamma(c)}{ }_{1} F_{1}(a ; c ;-x t) f(t) d t=\frac{g(x)}{\Gamma(a)} \text { for all } x>0,
$$

the integral being Lebesgue. The factor $t^{c-1}$, and the gamma functions, could of course be absorbed into the unknown function $f$.

THEOREM 12. Let re $a>0$, re $c>0$, and let $\mathrm{g}$ be the Laplace transform of a function $\mathscr{L}^{-1} g \in E$. Then

$$
f(x)=J^{a-c} x^{1-a} \mathscr{L}^{-1} g(x)
$$

is a solution of (18) in $E$ if either

(i) re $c>$ re $a$ and $x^{c-1} J^{a-c} x^{1-a} \mathscr{L}^{-1} g(x) \in L(0,1)$, or

(ii) re $a>$ re $c$, or

(iii) there is $k$ such that re $k>\max \{$ re $a$, re $c\}$ and

$$
x^{k-1} J^{a-k} x^{1-a} \mathscr{L}^{-1} g(x) \in L(0,1) .
$$

Further, under (i) this is the only solution of (18) in E; under (ii) it is the only solution of (18) in E satisfying

$$
x^{a-1} J^{c-a} f(x) \in L(0,1) ;
$$

under (iii) it is the only solution of (18) in E satisfying

$$
x^{k-1} J^{c-k} f(x) \in L(0,1) \text {. }
$$

Proof. It is easily verified, from definition and Leibniz's rule, that the product of a 
power with a function in $E$ is also in $E$; we use this fact frequently in this proof. In particular, $x^{1-a} \mathscr{L}^{-1} g(x) \in E$. By Theorem 2 or Lemma $5, f$ defined by (19) exists in $E$. gives

(i) Suppose re $c>$ re $a$ and (20) holds. Then $x^{c-1} f(x)$ is in $L(0,1)$, and Lemma 11(i)

$$
\begin{aligned}
\int_{0}^{\infty} \frac{t^{c-1}}{\Gamma(c)}{ }_{1} F_{1}(a ; c ;-x t) f(t) d t & =\frac{1}{\Gamma(a)} \mathscr{L} x^{a-1} J^{c-a} f(x) \\
& =\frac{1}{\Gamma(a)} \mathscr{L} x^{a-1} x^{1-a} \mathscr{L}^{-1} g(x)=\frac{g(x)}{\Gamma(a)},
\end{aligned}
$$

using (19) and Theorem 8 (case (i)). So $f$ is a solution of (18) in $E$.

If there were more than one solution of (18) in $E$, let $h$ be the difference of two of them; then $h \in E$ and

$$
\int_{0}^{\infty} t^{c-1}{ }_{1} F_{1}(a ; c ;-x t) h(t) d t=0, \text { for all } x>0 .
$$

Since the integrand is asymptotic to $t^{c-1} h(t)$ as $t \rightarrow 0$ and the integral is Lebesgue, $t^{c-1} h(t) \in L(0, \delta)$ for $\delta$ sufficiently small; and since $t^{c-1} h(t)$ is continuous in $(0,1]$, it is also in $L(0,1)$. So by Lemma 11(i), and (25), the Laplace transform of $t^{a-1} J^{c-a} h(t)$ vanishes. By [10: Theorem $6.3 \mathrm{p} .63]$ this function $t^{a-1} J^{c-a} h(t)$ is zero almost everywhere in $(0, \infty)$, and hence so is $J^{c-a} h(t)$. Being in $E$ by Theorem $2, J^{c-a} h(t)$ is zero everywhere.

By Theorem 6 and Theorem 8 (case (ii)),

$$
h(x)=J^{0} h(x)=J^{a-c} J^{c-a} h(x)=J^{a-c} 0(x),
$$

where 0 is the zero function. Let $n$ be an integer such that $n>\operatorname{re}(c-a)$. By (2), Lemma 5 and (1),

$$
h(x)=J^{a-c} 0(x)=(-D)^{n} J^{n+a-c} 0(x)=0
$$

for all $x>0$, which proves the uniqueness of solutions of (18) in $E$.

(ii) Suppose re $a>$ re $c$. By (19) and Theorem 8 (case (ii)),

$$
x^{a-1} J^{c-a} f(x)=x^{a-1} J^{c-a} J^{a-c} x^{1-a} \mathscr{L}^{-1} g(x)=x^{a-1} J^{0} x^{1-a} \mathscr{L}^{-1} g(x)=\mathscr{L}^{-1} g(x),
$$

using also Theorem 6. Now $\mathscr{L}^{-1} g \in L(0,1)$ since it has a Laplace transform; so, with (27), Lemma 11(ii) gives equations (24). Thus $f$ is a solution of (18) in $E$; and further $f$ satisfies (22).

Let $h$ be the difference of two solutions of (18) which are in $E$ and also satisfy (22). Then $h \in E$, (25) holds, and also $x^{a-1} J^{c-a} h(x) \in L(0,1)$. So by Lemma 11(ii), and (25), the Laplace transform of $t^{a-1} J^{c-a} h(t)$ vanishes. As in (i), $J^{c-a} h(t)$ is zero almost everywhere; and, being in $E$ by Lemma 5 , it is zero everywhere.

By Theorem 6 and Theorem 8 (case (i)), (26) holds. But since re $(a-c)>0$, the definition (1) gives that $J^{a-c} 0(x)=0$, and so by (26) $h(x)=0$. This proves the desired uniqueness.

(iii) Suppose there is $k$ such that re $k>\max \{$ re $a$, re $c\}$ and (21) holds. By (19) and 
Theorem 8,

$$
x^{k-1} J^{c-k} f(x)=x^{k-1} J^{c-k} J^{a-c} x^{1-a} \mathscr{L}^{-1} g(x)=x^{k-1} J^{a-k} x^{1-a} \mathscr{L}^{-1} g(x) \in L(0,1),
$$

using (21); so, by Lemma 11(iii), equations (24) hold. So $f$ is a solution of (18) in $E$; and further $f$ satisfies (23).

Let $h$ be the difference of two solutions of (18) which are in $E$ and also satisfy (23). Then $h \in E$, (25) holds, and also $x^{k-1} J^{c-k} h(x) \in L(0,1)$. By Lemma 11(iii), and (25), the Laplace transform of $t^{a-1} J^{c-a} h(t)$ vanishes. As in (i), $J^{c-a} h(t)$ is zero almost everywhere; and, being in $E$ by either Theorem 2 or Lemma 5 , it is zero everywhere.

Finally $h$ is proved to be the zero function, by the method of (i) associated with (26) if $\operatorname{re}(c-a) \geq 0$, and by the method of (ii) associated with (26) if $\operatorname{re}(c-a)<0$.

\section{REFERENCES}

1. A. Erdélyi, W. Magnus, F. Oberhettinger and F. G. Tricomi. Higher Transcendental Functions Vol. 1 (McGraw-Hill, 1953).

2. M. J. Lighthill, Fourier Analysis and Generalized Functions (Cambridge, 1958).

3. E. R. Love, Some integral equations involving hypergeometric functions, Proc. Edinburgh Math. Soc. (2) 15 (1967), 169-198.

4. E. R. Love, Two more hypergeometric integral equations, Proc. Cambridge Phil. Soc. 63 (1967), 1055-1076.

5. E. R. Love, A hypergeometric integral equation in Fractional Calculus and its Applications (edited by B. Ross) (Springer Lecture Notes No 457, 1974) 272-288.

6. K. S. Miller, The Weyl fractional calculus in Fractional Calculus and its Applications (edited by B. Ross) (Springer Lecture Notes No 457, 1974) 80-89.

7. T. R. Prabhakar, Two singular integral equations involving confluent hypergeometric functions, Proc. Cambridge Phil. Soc. 66 (1969), 71-89.

8. T. R. Prabhakar, Some integral equations with Kummer's function in the kernels, Canad. Math. Bull. 14 (1971), 391-404.

9. H. M. Srivastav and R. G. Buschman, Convolution Integral Equations with Special Function Kernels (Wiley Eastern, Delhi, 1976).

10. D. V. Widder, The Laplace Transform (Princeton, 1946).

E. R. Love

The University of Melbourne

ParkVILLE 3052

Australia
T. R. Prabhakar and N. K. Kashyap The UnIVERSITY of DeLhI Delhi 110007

INDIA 